\title{
The Young Stars in IC 1613
}

\section{J. Borissova}

Institute of Astronomy, Bulgarian Academy of Sciences, 72 Tsarigradsko chaussèe, BG-1784 Sofia, Bulgaria

L. Georgiev, M. Rosado and G. Koenigsberger

Instituto de Astronomía, Universidad Nacional Autónoma de México, México

R. Kurtev and G. Ivanov

Department of Astronomy, Sofia University, BG-1164 Sofia, Bulgaria

\begin{abstract}
Deep $U B V$ and $J H K$ photometry of the stars in IC 1613 was used to investigate the stellar content in the HII regions of the northeast sector of the galaxy. The ages of nine of the associations outlined by Hodge (1978) were determined. A10 and A14 were found to be the youngest associations with ages of approximately $5 \mathrm{Myr}$, while A12, A18 and A19 were the oldest ones found, having a common age of approx. 20 Myr. Analysis of theoretical HR diagrams shows the presence of young massive stars in the associations, which have been born almost coeval. We have also found evidence of a difference between the slopes of the IMFs and differential LFs, between the group of associations embedded in gas and the group containing A12, A18 and A19, which are not affected by nebulosity.
\end{abstract}

\section{Introduction}

The study of the $\mathrm{OB}$ associations in IC 1613 is motivated by the fact that they can provide information about star formation processes, evolution of massive stars and the interaction between stars and the interstellar medium.

\section{Observations and Data Reduction}

The $U B V$ observations of IC 1613 were carried out on the 2-m telescope of the Bulgarian National Astronomical Observatory. The observing area was a $5.6^{\prime} \times 5.6^{\prime}$ field centred on the H II regions in the northeast sector of the galaxy. An additional set of near-IR, $J H K$, images in the same field of IC 16131 was obtained with the Infrared Camera "Camila" attached to the 2.1-m telescope of the Observatorio Astronomico National, UNAM, Mexico. The standard IRAF data reduction packages were used to carry out the basic CCD reductions. The stellar photometry of the frames was done using DAOPHOT II (Stetson 1993). The 

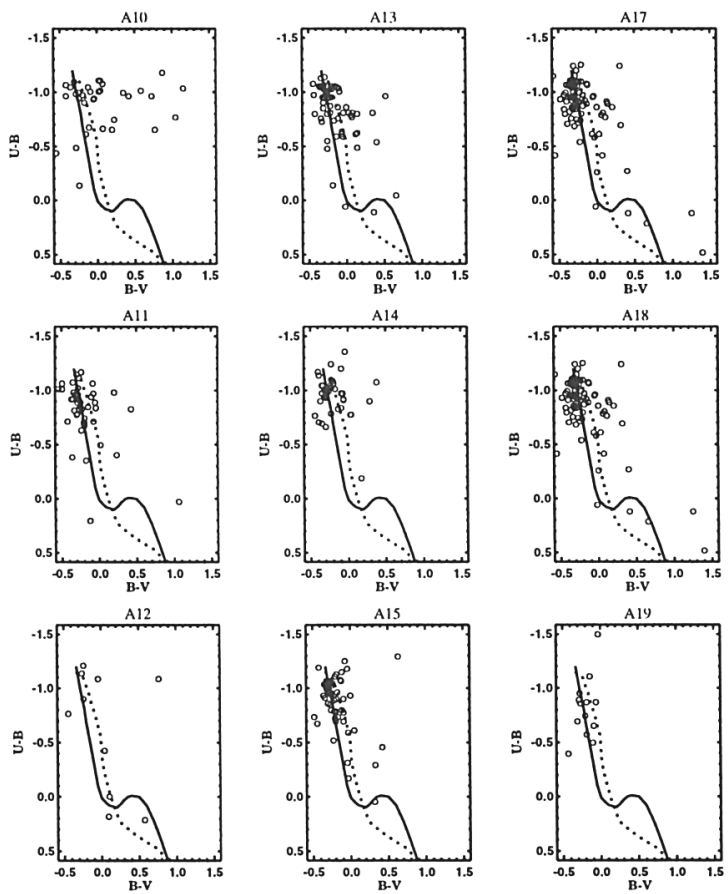

Figure 1. $(U-B, B-V)$ color-color diagrams obtained for Hodge associations. The fiducial locus of stars of luminosity class $\mathrm{V}$ and of luminosity class Iab (dashed line) are superimposed. The mean $E(B-V)=0.06$ was subtracted (Georgiev et al. 1998).

completeness limits are 21.5, 22.5, 22.5, 18.5, 18.0 and $18 \mathrm{mag}$ in the $U B V J H K$ filters, respectively. Only stars which had photometric errors no larger than 0.15 mag were used in our analysis.

\section{Associations}

In our field of observation we have nine of the twenty Hodge associations - Nos $10,11,12,13,14,15,17,18$ and 19 . The $(B-V, U-B)$ color-color diagrams (Fig. 1) show well defined main sequences with widths of typically 0.2 .

The ages of the associations were determined by means of an isochrone fit with the isochrones from the Padua library using Flannery \& Johnson's (1982) method. We chose for our comparison metallicity $\mathrm{z}=0.004$ (Kingsburgh \& Barlow 1995), distance modulus 24.20 (Freedman 1988) and the individual stellar reddening calculated by the $Q$ method. The "most probable" age of the associations is given in Table 1 . There are significant age differences which are generally larger than the uncertainty of the method (2.5 Myr). A10 and A14 were found to be the youngest associations with ages of approximately $5 \mathrm{Myr}$, while A12, A18 and A19 were the oldest ones found, having a common age of approximately $20 \mathrm{Myr}$. 
Table 1. Parameters of the Hodge associations

\begin{tabular}{lrrrrrrrr}
\hline Assoc. & $\begin{array}{r}\text { No. blue } \\
\text { stars }\end{array}$ & $\begin{array}{r}\text { Age } \\
(\mathrm{Myr})\end{array}$ & $\begin{array}{c}M_{u p} \\
\left(M_{\odot}\right)\end{array}$ & $\begin{array}{r}N_{12} \\
\left(M_{\odot}\right)\end{array}$ & Name & $U-B$ & $B-V$ & $\mathrm{~V}$ \\
\hline A10 & 27 & 5 & 60 & 14 & A43 & -0.60 & 0.12 & 16.57 \\
A10 & - & - & - & - & A42 & -0.95 & -0.19 & 18.33 \\
A10 & - & - & - & - & WR4 & -0.39 & -0.41 & 19.26 \\
A11 & 31 & 8 & 50 & 9 & $22 \mathrm{~A}$ & -0.98 & -0.21 & 17.46 \\
A12 & 6 & 20 & 25 & 3 & - & - & - & - \\
A13 & 58 & 10 & 40 & 28 & S13 & -0.56 & 0.00 & 18.12 \\
A14 & 44 & 5 & 60 & 27 & WR8 & -1.79 & 0.53 & 19.55 \\
A14 & - & - & - & - & $38 \mathrm{a}$ & -1.09 & -0.14 & 18.87 \\
A15 & 52 & 10 & 40 & 28 & - & - & - & - \\
A17 & 74 & 8 & 70 & 33 & B42 & -1.00 & -0.22 & 17.78 \\
A18 & 16 & 20 & 20 & 7 & - & - & - & - \\
A19 & 12 & 20 & 15 & 3 & - & - & - & - \\
& & & & & & & &
\end{tabular}

To plot the stars on the theoretical HR diagram we use the equations of Massey et al. (1995) for $U B V$ data and Costa \& Frogel (1996) for the $J H K$ system. The distance modulus, reddening and metallicity are as in the isochrone comparison. The evolutionary mass tracks from Charbonel et al. (1993) were superimposed. The masses of the most massive stars are listed in column 4 of Table 1 and column 5 contains a number of stars with masses higher than $12 M_{\odot}$ (denoted $N_{12}$ ). The analysis of Table 1 shows that the associations containing the most massive stars are the youngest ones while the associations containing only low-mass stars are indeed the oldest.

It is not possible to calculate the IMF for each association because of the small numbers of association members, leading to large statistical errors. To avoid this we combined the Hodge associations in three groups. The first group contains the blue stars from A10, A11, A14 and A17 (having ages between 5-8 $\mathrm{Myr}$ ), the second group contains A13 and A15 (having age $10 \mathrm{Myr}$ ) and the last one contains the stars from A12, A18 and A19 (with an age of $20 \mathrm{Myr}$ ). According to Fabry Pérot images (Rosado, 1998 private communication) the stars from the first and the second group are embedded in shells of gas, while there is no gas at all in the last association group (A12, A18 and A19). The slopes of the IMFs for these three groups are $\Gamma=-1.59 \pm 0.18, \Gamma=-2.22 \pm 0.20$ and $\Gamma=-2.65 \pm 0.05$ respectively (the uncertainty is the least-squares fit error). It is not a strong and statistically significant effect for groups I and II and groups II and III, but becomes more significant for groups I and III (approx. 6 $\sigma$ ).

The differential luminosity function (LF) is an important quantity because differences in stellar population should be reflected in the LF. For the same reason as in the case of the IMF (few association members and poor statistics) we calculated the slope of the LF for the above-defined three groups of associations in $M_{V}$, including only blue stars $((U-V)<-0.5)$. The slopes of the LFs for these three groups are $\alpha=0.48 \pm 0.07, \alpha=0.32 \pm 0.06$ and $\alpha=0.26 \pm 0.05$ respectively (the uncertainty is the least-squares fit error).

The different stellar content, age differences, variations of slopes of IMFs, variations of slopes of differential LFs and the presence of gas in some of the associations, show a signature of a presence of two waves of star formation. It is possible however that the differences are not physical and are due to an observational effect because of the observational limitations. 


\section{Bright Stars in the Associations}

Using various photometric criteria $(U-B, B-V, J-H, H-K$ colors, the reddening independent $Q$ parameter, $M_{V}$ and $M_{K}$ absolute magnitudes and positions in the $M_{b o l}, \log \left(T_{e f f}\right)$ diagrams) we selected six possible blue supergiants and seven possible red supergiants. Five of the blue supergiants and three of the red supergiants are proposed by Sandage \& Katem (1976) and confirmed by Humphreys (1980). Three Wolf-Rayet (WR) stars were reported by Armandroff \& Massey (1985) in our field of view. Hodge (1978) included the brightest red supergiants V32 and V38 in the boundaries of A10 and A14. Table 1 contains colors and magnitudes of the blue supergiants and WR stars lying within the boundaries of the associations. The ratio between the blue and red supergiants and the WR stars in this most active area of IC 1613 is 5:7:3. If we compare the number of unevolved main sequence stars with masses larger than $40 M_{\odot}$ to the number of WR stars, we obtain the ratio 10:3, which is in excellent agreement with results of Massey (1995) for NGC 6822, M31, M33 and the LMC. The relative numbers of blue, red and WR stars in the associations may be an indicator of the times spent in different evolutionary stages, however our statistics for bright stars is not sufficient to make any definitive conclusions.

Acknowledgements. This research was supported by the "Programa de cooperations Mexico- Republica de Bulgaria - "Estrellas Masivas en las Galaxias del Grupo Local" Of. No. 403. S.T.A.C.R.I.25/97 and by the Bulgarian National Science Foundation grant under contract No. F-804/1998 with the Bulgarian Ministry of Education and Sciences. This work was performed while J.B. was a visiting astronomer in UNAM, Mexico under contacts CONACYT No.4003545-2398PE and DGAPA INI04696.

\section{References}

Armandroff, T., Massey, P. 1985, ApJ, 291, 685

Charbonnel, C., Maynet, G., Maeder, A., et al. 1993, A\&AS, 101, 415

Costa, E., Frogel, J. 1996, AJ, 112, 2607

Flannery, B., Johnson, B. 1982, ApJ, 263, 166

Freedman, W. 1988, AJ, 96, 1248

Georgiev, L., Borissova, J., Rosado, M. et al. 1998, A\&AS, 133, 1

Hodge, P. 1978, ApJS, 37, 145

Humphreys, R. 1980, ApJ, 238, 65

Kingsburgh, R.L., Barlow, M.J. 1995, A\&A, 295, 171

Massey, P., Lang, C., DeGioia-Eastwood, K., Garmany, C. 1995, ApJ, 438, 188

Massey, P. 1996, in: WR stars in the framework of stellar evolution, 33-rd Liége

Int. Astrophs. Coll., p. 361

Sandage, A., Katem, B. 1976, AJ, 81, 743

Stetson, P. 1993, Users manual for DAOPHOT II. 